\title{
Hipertensão arterial em idosos: prevalência e fatores associados no município de Três Lagoas/MS
}

\author{
Patrícia Rodrigues Souza*, Suse Barbosa Castilho**, Sônia Regina Jurado***
}

\begin{abstract}
${ }^{*}$ Enfermeira, Universidade Federal de Mato Grosso do Sul, Campus de Três Lagoas, MS, Especialista em Enfermagem do Trabalho pela UNIDERP - Universidade para o Desenvolvimento da Região do Pantanal, Pólo de Três Lagoas/MS, **Enfermeira, Universidade Federal de Mato Grosso do Sul, Campus de Três Lagoas/MS, ***Docente, Professora Adjunta, Chefe de Departamento, Universidade Federal de Mato Grosso do Sul, Departamento de Enfermagem e Biotecnologia Aplicada à Saúde, Campus de Três Lagoas/MS
\end{abstract}

\section{Resumo}

O Brasil carece de números precisos de incidência e prevalência de hipertensão arterial, diabetes e fatores de risco associados a essas patologias, principalmente na populaçáo idosa. Portanto, verifica-se a importância de estudos sobre o tema, devido ao significativo aumento deste segmento populacional no país. O presente trabalho teve como escopo levantar a prevalência da hipertensão arterial e diabetes e de fatores de risco associados a essas doenças na população idosa do município de Três Lagoas, Estado de Mato Grosso do Sul, Brasil. Neste estudo, foram estudados 117 idosos de ambos os sexos, os quais apresentaram índice de massa corpórea igual a 26,84 $\pm 0,44 \mathrm{~kg} / \mathrm{m}^{2}$; circunferência abdominal 95,65 $\pm 1,22 \mathrm{~cm}$; pressão arterial sistólica (PAS) e diastólica (PAD) igual a 130,09 $\pm 2,08$ e 78,88 $\pm 1,15 \mathrm{mmHg}$, respectivamente e níveis glicêmicos de 128,50 \pm 4,68 mg/dl. Do total de idosos estudados, 38,46\% eram hipertensos e 10,25\% apresentaram níveis glicêmicos acima da normalidade. Os níveis pressóricos correlacionaram-se significativamente com peso corporal, circunferência abdominal e índice de massa corpórea $(\mathrm{p}=0,0032, \mathrm{p}=0,0003$ e $\mathrm{p}=0,0168$, respectivamente) e os níveis glicêmicos correlacionaram-se significativamente com a ingesta de carboidratos $(\mathrm{p}=0,0151)$. O presente trabalho mostrou, em síntese, que a hipertensão arterial e diabetes são mais prevalentes em idosos do que na população em geral.

Palavras-chave: hipertensão, diabetes, prevenção, idosos.

\section{Abstract \\ Arterial hypertension in the elderly: prevalence and associated factors in the city of Três Lagoas/MS}

Brazil has a lack of accurate figures regarding the incidence and prevalence of arterial hypertension, diabetes and risk factors associated with these diseases, especially in the elderly population. Therefore, it confirms the importance of studies on the subject, due to the significant increase of older people. This work aimed to assess the prevalence of hypertension and associated risk factors in the elderly population in the city of Três Lagoas/MS. The study comprised 117 elderly of both gender, who 
had body mass index $26.84 \pm 0.44 \mathrm{~kg} / \mathrm{m}^{2}$, waist circumference $95.65 \pm 1.22 \mathrm{~cm}$; systolic blood pressure (SBP) and diastolic (DBP) $130.09 \pm 2.08$ and $78.88 \pm 1.15 \mathrm{mmHg}$, respectively, and glucose levels of $128.50 \pm 4.68 \mathrm{mg} / \mathrm{dl} .38 .46 \%$ of the total number of elderly were hypertensive and $10.25 \%$ had blood glucose levels above normal. Pressure levels were significantly correlated with body weight, waist circumference and body mass index $(\mathrm{p}=0.0032, \mathrm{p}=0.0003$ and $\mathrm{p}=0.0168$, respectively) and glucose levels were significantly correlated with carbohydrate intake $(p=0.0151)$. Concluding, this study showed that hypertension and diabetes are more common in the elderly than in the general population.

Key-words: hypertension, diabetes, prevention, aged.

\section{Resumen}

\section{Hipertensión arterial en personas mayores: prevalencia y factores asociados en el municipio de Três Lagoas/MS}

El Brasil carece de números exactos de incidencia y prevalencia de hipertensión arterial, diabetes y factores de riesgo asociados a padecer de esas patologías, principalmente en la población de personas mayores. Por tanto, se verifica la necesidad de estudios sobre el tema, debido al significativo aumento de personas mayores en la población del país. El objetivo de este estudio fue estimar la prevalencia de la hipertensión arterial y de la diabetes y factores de riesgo asociados a esas enfermedades en la población de personas mayores del municipio de Três Lagoas, Estado de Mato Grosso do Sul. Fueron estudiadas 117 personas mayores de ambos sexos, las cuales presentaron un índice de masa corporal igual a 26,84 $\pm 0,44 \mathrm{~kg} / \mathrm{m}^{2}$; circunferencia abdominal 95,65 $\pm 1,22 \mathrm{~cm}$; presión arterial sistólica (PAS) y diastólica (PAD) igual a 130,09 $\pm 2,08$ y 78,88 $\pm 1,15 \mathrm{mmHg}$, respectivamente y niveles glicémicos de 128,50 \pm 4,68 mg/dl. Del total de mayores estudiados, 38,46\% eran hipertensos y 10,25\% presentaron niveles glicémicos por encima del límite establecido. Los niveles presóricos se correlacionaron significativamente con peso corporal, circunferencia abdominal e índice de masa corporal ( $\mathrm{p}=0,0032, \mathrm{p}=0,0003$ e p =0,0168, respectivamente) y los niveles glicémicos se correlacionaron significativamente con la ingesta de carbohidratos ( $\mathrm{p}=0,0151)$. Se concluye que la hipertensión arterial y diabetes son más comunes en personas mayores que en la población en general.

Palabras-clave: hipertensión, diabetes, prevención, anciano.

\section{Introdução}

O envelhecimento é um desafio do mundo atual que afeta países ricos e pobres. Estima-se que cerca de um milhão de pessoas cruzam a barreira dos 60 anos de idade a cada mês, em todo o mundo [1]. Do ponto de vista demográfico, segundo Carvalho e Andrade [2], no plano individual envelhecer significa aumentar o número de anos vividos. Paralelamente à evoluçáo cronológica, coexistem fenômenos de natureza biopsíquica e social, importantes para a percepçáo da idade e do envelhecimento.

O maior desenvolvimento científico e tecnológico também tem favorecido o aumento da expectativa de vida. Por outro lado, este fato contribui para o maior risco no desenvolvimento de doenças crônico-degenerativas, destacando-se as cardiovasculares, que se constituem na principal causa de morte no Brasil [3].

A hipertensão arterial (HA), conhecida como pressão alta, é um das doenças crônico-degenerativas que representam grandes desafios para a saúde mundial. No Brasil, estima-se que $22 \%$ da população adulta sofra de hipertensão [4]. Metade dos portadores de hipertensão arterial desconhece a existência da doença, por não apresentar sintomas e não procurar serviço médico.

O diabetes mellitus (DM) constitui, ainda, uma das principais causas de insuficiência renal, amputação de membros inferiores e cegueira [4] e, é de grande interesse a promoção de medidas preventivas, pois constitui em um aspecto central para a manutenção e recuperação da saúde do idoso.

A hipertensão arterial e o diabetes mellitus constituem os principais fatores de risco populacional para as doenças cardiovasculares, motivo pelo qual representam agravos de Saúde Pública, dos quais cerca de 60 a $80 \%$ dos casos podem ser tratados na rede básica [5].

Segundo Ponzetto[6], fatores de risco podem ser definidos como condiçóes que predispóem a um maior risco de desenvolver certo evento relacionado à saúde, após determinado tempo.

Para que se possam propor ações efetivas em saúde, em especial de enfermagem, aos idosos com diabetes e hipertensão arterial, faz-se necessário conhecer o estilo de vida desta população, bem como suas especificidades quanto à qualidade de 
vida. O acompanhamento e o controle do DM e da hipertensão no âmbito da atenção básica poderão evitar o surgimento e a progressão das complicaçóes, reduzindo o número de internaçôes hospitalares, bem como a mortalidade [5].

Este trabalho objetivou levantar a prevalência de diabetes, hipertensão arterial e de fatores de risco associados a essas patologias na população idosa participante de Núcleos Sociais da Terceira Idade no município de Três Lagoas/MS.

\section{Material e métodos}

A pesquisa foi desenvolvida com idosos em 16 núcleos sociais da Terceira Idade na cidade de Três Lagoas (MS), vinculados ao Departamento de Cidadania da Prefeitura Municipal local, por ocasião da coleta de dados, constituindo a amostra populacional para este estudo de 117 idosos. Os critérios de inclusão para constituir a amostragem foram: ter 60 anos ou mais e aderir à pesquisa. Os dados foram coletados no período de maio a outubro de 2008, utilizando-se de instrumento estruturado, avaliação de dados antropométricos e aferição de pressão arterial e níveis glicêmicos.

Os participantes foram informados do estudo a ser realizado e de sua importância para a saúde, por meio de palestra educativa, distribuição de folhetos explicativos sobre HA e DM. Após os esclarecimentos e de acordo com a vontade deles de participarem do estudo, assinaram o Termo de Consentimento Livre e Esclarecido - TCLE. Em seguida, foram entrevistados através de um questionário padronizado, abordando dados pessoais, tais como: hereditariedade, tabagismo, etilismo, sedentarismo, diabetes, raça, sexo, seguido de aferição da pressão arterial (PA), níveis de glicemia capilar, peso corporal, altura, circunferência abdominal e índice de massa corpórea (IMC). Durante a aferição da PA foi utilizado um esfigmomanômetro, marca $\mathrm{BIC}^{\oplus} \mathrm{e}$, para a avaliação dos níveis glicêmicos, utilizou-se um glicosímetro, marca Biocheck ${ }^{\oplus}$ TD-4225.

Os valores de PA obtidos foram analisados com base em algumas normas do V Consenso Brasileiro de Hipertensão Arterial [7] e os níveis glicêmicos foram preconizados de acordo com o Plano de Reorganização da Atenção à Hipertensão Arterial e ao Diabetes mellitus [4].

Os níveis glicêmicos e os níveis pressóricos foram correlacionados com peso corporal, circunferência abdominal, índice de massa corpórea, há- bitos alimentares, histórico familiar de hipertensão arterial e diabetes mellitus e, práticas de exercícios físicos. Foi utilizada a correlação de Pearson e em todos os testes estatísticos o índice de significância considerado foi de 0,05 .

\section{Resultados e discussão}

Foram coletados dados de 117 indivíduos com idade igual ou superior a 60 anos, variando entre 60 - 96 anos. Os maiores percentuais foram para os sujeitos do sexo feminino correspondendo a 58,12\% (68), raça branca 54,70\% (64) e parda com 24,78\% (29) dos entrevistados. Em relação ao estado civil, a maioria eram viúvos $(34,19 \%)$ ou casados formalmente $(33,33 \%)$ e apenas $15,38 \%$ (18) eram solteiros, por conseguinte, $87,18 \%$ (102) possuíam filhos, variando entre cinco filhos ou mais (25,64\%). Quanto à religião, 83,76\% (98) verbalizaram seguir ao catolicismo e, somente 7,69\% (09) ao protestantismo.

Da amostra estudada, 53\% tinham instrução de oito anos ou menos de estudo formal, 09 dos entrevistados $(7,69 \%)$ não eram alfabetizados e, $10,25 \%$ (12) concluíram o ensino superior.

Os dados antropométricos, os níveis pressóricos e glicêmicos dos idosos estão ilustrados na Tabela I.

Tabela I - Dados antropométricos, níveis pressóricos e glicêmicos dos idosos do município de Três Lagoas/MS, 2008.

\begin{tabular}{lll}
\hline Parâmetro & Média & DP \\
\hline Índice de Massa Corpórea (IMC) & 26,84 & 0,44 \\
Pressão Arterial Sistólica (mmHg) & 130,09 & 2,08 \\
Pressão Arterial Diastólica (mmHg) & 78,88 & 1,15 \\
Peso corporal (kg) & 68,79 & 1,42 \\
Glicemia (mg/dl) & 128,50 & 4,68 \\
Altura (cm) & 154,77 & 2,82 \\
Circunferência abdominal $(\mathrm{cm})$ & 95,65 & 1,22 \\
\hline
\end{tabular}

Dos 117 participantes, 20,51\% (24) autoreferiram ter diagnóstico de diabetes mellitus tipo II e, entre esses 24 diabéticos, 17 eram portadores de hipertensão arterial. Estes dados corroboram informaçóes abordadas pelo Ministério da Saúde [5], quando afirmam que o DM tipo II é, hoje, considerado parte da chamada síndrome plurimetabólica ou de resistência à insulina e ocorre em $90 \%$ dos pacientes diabéticos, aumentando a prevalência com a idade, podendo chegar a $20 \%$ na população com 60 anos ou mais. $\mathrm{O}$ índice de diabéticos 
confirmados pelo exame de glicemia no estudo foi de $10,25 \%$, ao passo que na população adulta este valor é de 7,6\% [5].

O índice de prevalência de hipertensão, estimada pelo Systolic Hypertension in the Elderly Program - SHEP, variou em torno de 5\% nos idosos com idade entre 60 a 69 anos, em cerca de $10 \%$ na faixa acima de 70 anos e $20 \%$ naqueles com mais de 80 anos [8-10]. Em nosso estudo, encontramos um total de 38,46\% de hipertensos, o que corresponde a quase o dobro de hipertensos (20\%) encontrados na população adulta [5]. Seguindo a faixa etária dos hipertensos, os achados no presente estudo foram os seguintes: $53,33 \%$ de 60 a 69 anos; $26,66 \%$ de 70 a 79 anos e $4,41 \%$ acima de 80 anos. Salientamos que os valores de hipertensos encontrados em nosso estudo nas faixas etárias de 60-69 anos e 70-79 anos estão acima dos valores encontrados em outros trabalhos.

O Estudo Epidemiológico Longitudinal com Idoso - Epidoso - de 1991, seguindo os critérios dos Consensos Brasileiro e Americano de Hipertensão, encontrou, entre 1.667 idosos, uma prevalência de $\mathrm{HA}$ em torno de $63 \%$ nas mulheres e de $57 \%$ nos homens [11]. Em nosso estudo foi encontrada uma maior prevalência de hipertensão arterial nos homens $(19,65 \%)$ do que nas mulheres $(18,80 \%)$, mostrando valores inferiores do estudo acima citado.

Os níveis pressóricos correlacionaram-se significativamente com peso corporal, circunferência abdominal e IMC ( $\mathrm{p}=0,0032, \mathrm{p}=0,0003, \mathrm{p}=$ $0,0168)$. Sabe-se que indivíduos obesos são mais predispostos à ocorrência de eventos cardiovasculares e maiores níveis de pressão arterial que indivíduos com peso normal [12], o que corrobora com nossos achados. Um total de 37,60\% (44) dos idosos estudados declararam possuir doença cardiovascular, cerebrovascular ou vascular periférica definida.

Os idosos são um segmento populacional que merece maior atenção com relação aos fatores causais e de riscos associados à hipertensão e diabetes. Em um estudo realizado por Passos et al. [13] foi demonstrado que um grupo de idosos apresentou maiores taxas de prevalência de inatividade física, tabagismo, hipertensão arterial, níveis de colesterol total e triglicerídeos do que o grupo de adultos.

Em continuação aos resultados obtidos por Passos et al. [13], o diabetes foi estatisticamente associado com menor escolaridade e renda, tabagismo, uso de bebidas alcoólicas, elevado índice de massa corporal, presença de hipertensão arterial sistêmica, história familiar de diabetes, contudo, em nosso estudo não obtivemos correlações significativas entre os níveis glicêmicos e os parâmetros acima descritos.

Os níveis glicêmicos foram maiores nas mulheres $(58,15 \%)$ do que nos homens $(41,85 \%)$. A glicemia correlacionou-se significativamente com a ingesta de carboidratos nos idosos neste estudo ( $\mathrm{p}$ $=0,0151$ ). Portanto, existe uma alteração no metabolismo de carboidratos ingeridos. Náo se sabe se essa hiperglicemia é normal ou patológica, contudo, necessita de intervençóes terapêuticas. A causa das alteraçóes no metabolismo dos carboidratos relacionadas à idade ainda está em discussão. Algumas possibilidades estâo relacionadas à dieta insuficiente, inatividade física, diminuição da massa magra corporal, secreçáo alterada de insulina e resistência à glicose [14].

De acordo com o Ministério da Saúde [4], cerca de $50 \%$ da população com diabetes náo sabe que são portadores da doença, algumas vezes permanecendo náo diagnosticados até que se manifestem sinais de complicaçôes. Por isso, testes de rastreamento são indicados em indivíduos assintomáticos que apresentem maior risco da doença.

\section{Conclusão}

O presente trabalho mostrou que a hipertensão arterial e o diabetes são mais prevalentes em idosos do que na população adulta. Ainda que as políticas públicas devam contemplar a todos, atenção especial deve ser voltada para a população idosa, tanto para as açóes de prevenção, de controle da hipertensão, assim como para as de promoção à saúde.

\section{Agradecimentos}

À Pró-Reitoria de Extensão e Assuntos Estudantis (PREAE) da Universidade Federal de Mato Grosso do Sul pelo apoio financeiro na execução do projeto.

\section{Referências}

1. Veras RP, Lourenço R, Martins CSF, Sanchez MAS, Chaves PH. Novos paradigmas do modelo assistencial no setor saúde: conseqüência da explosão populacional dos idosos no Brasil. In: Veras RP. Terceira idade: gestão contemporânea em saúde. Rio de Janeiro: Relume Dumará; 2002. p. 11-79.

2. Instituto Brasileiro de Geografia e Estatística - IBGE. Ministério do Planejamento, Orçamento e Gestão, Diretoria de Pesquisas, Departamento de População 
e Indicadores Sociais. Perfil dos idosos responsáveis pelos domicílios no Brasil 2000/ Ministério do Planejamento, Orçamento e Gestão, Diretoria de Pesquisas, Departamento de População e Indicadores Sociais. Rio de Janeiro: Ministério do Planejamento, Orçamento e Gestão; 2002. 97p.

3. Martins LM, França APD, Kimura M. Qualidade de vida de pessoas com doença crônica. Rev Latinoam Enfermagem 1996;4(3):5-18.

4. Brasil. Ministério da Saúde, Secretaria de Atenção à Saúde, Departamento de Atenção Básica. Envelhecimento e saúde da pessoa idosa / Ministério da Saúde, Secretaria de Atenção à Saúde, Departamento de Atenção Básica. Brasília: Ministério da Saúde; 2006.

5. Brasil. Ministério da Saúde, Secretaria de Políticas de Saúde, Departamento de Açôes Programáticas Estratégicas. Plano de reorganização da atenção à hipertensão arterial e ao diabetes mellitus. Brasília: Ministério da Saúde; 2001.

6. Ponzetto M, Maero B, Maina P, D'agostino E, Scarafiotti C, Speme S, et al. Risk factors in the elderly. Arch Gerontol Geriatr 2002;(8 Suppl):283-90.

7. World Health Organization. Envelhecimento ativo: uma política de saúde. Traduzido por: Suzana Gontijo. Brasília: Organização Pan-Americana da Saúde; 2005. 60p.

8. Morigutti JC, Paiva CE, Marchini JS, Furtado Júnior DA, Matos FD, Ferriolli E. Systolic hypertension in elderly program e outros estudos clínicos em idosos. Rev Bras Hipertens 2001;2:206-11.
9. Mulrow CD, Brand MB. Hipertensão arterial no idoso. In: Gallo JJ, Whitehead JB, Rabins PV, Silliman RA, Murphy JB, eds. Reichel assistência ao idoso: aspectos clínicos do envelhecimento. Rio de Janeiro: Guanabra Koogan; 1999. p. 99-08.

10. Shep - Cooperative Research Group. Prevention of stroke by antihypertensive drug treatment in older persons with isolated systolic hypertension: final results of the systolic hypertension in the elderly program (SHEP). JAMA 1991;265:3255-64.

11. Ramos LR, Toniolo J, Cendoroglo M, Garcia JT, Najas MS, Perracini M, et al. Two-years follow-up study of elderly residents in São Paulo, Brazil (Epidoso Project): methodology and preliminary results. Rev Saúde Pública 1998;5:397-07.

12. Carneiro G, Faria NA, Ribeiro Filho FF, Guimarães A, Lerário D, Ferreira SRG, et al. Influência da distribuição da gordura corporal sobre a prevalência de hipertensão arterial e outros fatores de risco cardiovascular em indivíduos obesos. Rev Assoc Med Bras 2003;49(3):306-11.

13. Passos VMA, Barreto SM, Diniz LM, Lima-Costa MF. Type 2 diabetes: prevalence and associated factors in a Brazilian community - the Bambuí health and aging study. São Paulo Med J 2005;123(2):66-71.

14. Smeltzer SC, Bare BG. Brunner \& Suddarth's: tratado de enfermagem médico-cirúrgica. $10^{\mathrm{a}} \mathrm{ed}$. Rio de Janeiro: Guanabara Koogan; 2006. p.1215-73. 